\title{
10-year survival of a patient with metastatic prostate cancer: Case report and literature review
}

\author{
Kristian Krpina, Dean Markić, Dražen Rahelić, Juraj Ahel, Nino Rubinić, Josip Španjol \\ Department of Urology, Clinical Hospital Center Rijeka, Rijeka, Croatia.
}

\begin{abstract}
Summary Prostate cancer is the most common malignancy in men. The 5-year relative survival for all stages combined is $98.8 \%$. Patients diagnosed with metastatic prostate cancer have median survival from 2 to 3 years. We describe a case of 64-year old man who clinically presented with inguinal lymphadenopathy. Because of elevated PSA levels biopsy of prostate was done and adenocarcinoma was diagnosed. Biopsy of inguinal lymph nodes confirmed the diagnosis of prostate cancer. Hormonal treatment was started and at the most recent follow-up, 10 years later, the patient is asymptomatic with no clinical signs of disseminated disease.
\end{abstract}

KEY WORDS: Generalized lymphadenopathy; Prostate cancer: Survival: Treatment.

Submitted 1 February 2015; Accepted 31 March 2015

\section{INTRODUCTION}

Prostate cancer is the most common malignancy in men. The median age at diagnosis of prostate cancer is 68 years. Prostate cancer does not alter life expectancy for most of these men as the 5-year relative survival for all stages combined is $98.8 \%$. Those who present with metastatic disease have a median survival of approximately 30 months, however a small proportion of men diagnosed with metastatic prostate cancer will live substantially longer. One of the rare presentations of metastatic prostate cancer are generalized lymphatic metastases (1). We report a case of prostate cancer patient who clinically manifested as generalized lymphadenopathy with concomitant minimal bone metastases and subsequent ten years follow up.

\section{Case report}

In March 2004, a 64-year-old male was referred to Clinical Hospital Center Rijeka for a constipation. The patient reported swelling of the left leg and suprapubic fullness. He denied any voiding difficulties. The patient's past medical and surgical history were not contributory. Physical examination of the patient showed palpabile suprapubic fullness, enlarged lymph nodes in left inguinum and left leg oedema. Digital rectal examination (DRE) revealed nodular induration in the lateral lobes of the prostate which partially ocluded the rectum. PSA level was $152 \mathrm{ng} / \mathrm{mL}$. Transrectal ultrasound (TRUS) showed prostatic enlargement with multiple hypoechoic peripheral lesions. Chest X ray showed no pathological findings. Computed tomography (CT) of the abdomen and pelvis was remarkable for an enlarged retroperitoneal and inguinal lymph nodes with dislocation of the left ureter and bladder to the right side (Figure 1). Cystography showed bladder dislocated to the projection of the right iliac bone. The bone scan showed metastasis in the fifth thoracic vertebra. Transrectal biopsy of the prostate demonstrated adenocarcinoma (Gleason score 5+5, "signet ring"). Biopsy of the left inguinal lymph node also revealed adenocarcinoma with immunohistochemical staining that was strongly positive for PSA. The patient underwent castration and therapy was continued with flutamide. Afterwards, during time, his PSA level reached $0.16 \mathrm{ng} / \mathrm{mL}$. In 2008, the patient was lost to follow-up. He was again referred to urology consultant in December 2012. The patient stated that he stopped talking medications in 2008, because of the adverse effects. His PSA level was $3.8 \mathrm{ng} / \mathrm{mL}$ and bicalutamide treatment was started. At the most recent check-up in September 2014, CT of the abdomen and pelvis showed no evidence of disease. His PSA level is $0.32 \mathrm{ng} / \mathrm{mL}$ and he is asymptomatic.

\section{Discussion}

Despite advances in the treatment of prostate cancer, nearly 260,000 men worldwide will die from the disease this year, and the vast majority will have metastatic disease at the time of death. While in patients with metastatic prostate cancer median survival is approximately 30 months when treated with hormonal therapy, there is substantial inter patient variation. Why this disease has such a divergent response to hormonal therapy is a fundamental question of urologic oncology. Given the heterogeneity of prognoses in patients with metastatic prostate cancer, the ability to accurately predict survival is vital for optimal patient counseling, selection of treatments, clinical trial design, and interpretation of clinical data. Over the past 20 years, several prognostic models have been developed in an attempt to refine the clinician's predictive ability.

Glass et al. through their clinical data analysis identified 
four prognostic factors which have a major impact on outcome of metastatic prostate cancer patients (2).

Namely those are appendicular versus axial disease, performance status 0 versus 1 to 3, PSA less than 65 versus $65 \mathrm{ng} / \mathrm{ml}$ or greater and Gleason score less than 8 versus 8 or greater. Using these criteria three prognostic groups can be made, including a good, intermediate and poor group. For example, estimation of five-year survival in the poor prognostic group is only $9 \%$. Authors concluded that risk stratification according to these factors is helpful for individual patient treatment.

The EORTC conducted two randomized phase III trials (trial 30,843 and trial 30,853) of maximal androgen blockade in 695 patients with metastatic prostate cancer. These trials showed discrepancy of results according to overall survival of treated patients. In order to determine whether differences in patient characteristics could explain these possibly contradictory results, Sylvester at al used a statistical model to identify prognostic factors for survival. Their model revealed six prognostic factors such as alkaline phosphatase, hemoglobin, performance status, pain score, $\mathrm{T}$ category and G grade. By using these factors patients can be divided into two risk groups (good or poor prognosis with 3.5 and 1.75 years' median survival, respectively). In conclusion, authors underline the importance of taking into account patient characteristics when treatment strategy is planned.

Montgomery study revealed higher body mass index to be associated with better overall and progression-free survival in patients with androgen dependent metastatic prostate cancer. The question remains what is real clinical application of these study-results in every day clinical setting. On example of our case-presentation it is possible to see that the same patient would be grouped differently according to different authors. For example, Glass criteria would group the presented patient in poor prognostic group whereas Sylvester model would group him in good prognosis group.

On the other hand one of the peculiarities of this case is clinical presentation. Although many cases have been reported of prostate cancer metastasizing to inguinal nodes many years after diagnosis only one case has been reported in which inguinal lymphadenopathy was the presenting sign (3). The patient presented here had not undergone any previous local surgery that might have distorted the lymphatic drainage of the prostate.
Figure 1.

CT of the abdomen showing enlarged retroperitoneal lymphnodes.

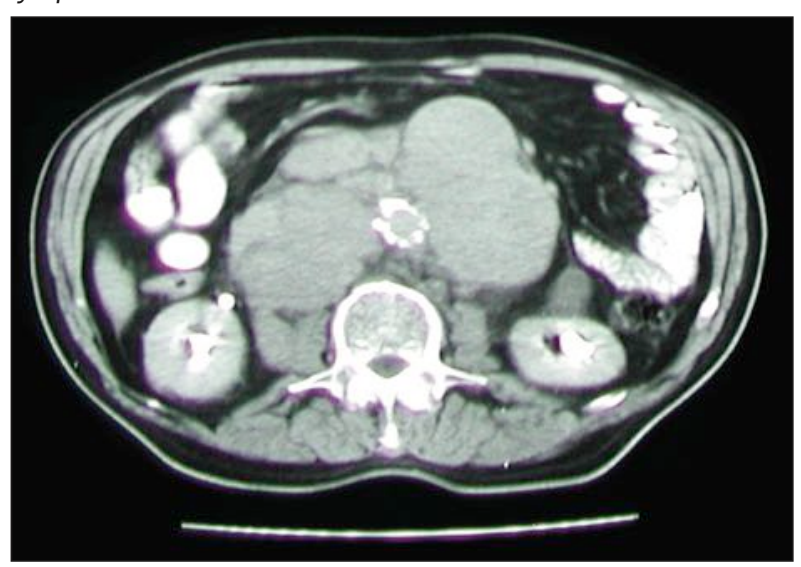

One possible explanation of such metastasis is that he might have had some aberrant lymphatic drainage of the prostate. Uncommon routes of pelvic lymphatic drainage that have been reported include the gonadal vessels, mesenteric and mesocolic nodes, posterior iliac crest nodes and inferior phrenic nodes. As prostate cancer specific mortality has fallen to low levels for many men prostate cancer is a chronic disease. Survivorship research has defined many risks and factors unique to this population but further work to identify novel prognostic markers is needed. Current efforts to better classify the molecular phenotypes of metastatic clones of cells may provide essential information in predicting the clinical behaviour of the disease.

\section{REFERENCES}

1. Moura FM, Garcia LT, Castro LPF, Ferrari TCA. Prostate adenocarcinoma manifesting as generalized lymphadenopathy. Urol Oncol. 2006; 24:216-9.

2. Glass TR, Tangen CM, Crawford ED, Thompson I. Metastatic carcinoma of the prostate: identifying prognostic groups using recursive partitioning. J Urol. 2003; 169:164-9.

3. Slavis SA, Golji H, Miller JB. Carcinoma of the prostate presenting as inguinal adenopathy. Cleve Clin J Med. 1990; 57:97.

\section{Correspondence}

Kristian Krpina, MD, PhD, FEBU (Corresponding Author) kristiank@net.hr

Dean Markić, MD, PhD, FEBU

Dražen Rahelić, MD

Juraj Ahel, MD

Nino Rubinic, MD

Josip Španjol, MD, PhD

Department of Urology, Clinical Hospital Center Rijeka

Tome Strižića 3, 51000 Rijeka, Croatia 